Research Article

\title{
Controlling the Emission Properties of Quantum Rods via Multiscale 3D Ordered Organization
}

\author{
Thomas Bizien $\left(\mathbb{D},{ }^{1,2,3}\right.$ Marie Postic, ${ }^{1,2}$ Pascale Even-Hernandez $\mathbb{D D}^{2}$ Pascal Panizza $\left(\mathbb{D},{ }^{1}\right.$ \\ Cristelle Mériadec, ${ }^{1}$ Florian Meneau $\mathbb{D}^{3,4}{ }^{3,4}$ Elsa Mazari-Arrighi, ${ }^{5}$ Christophe Dupuis, ${ }^{5}$ \\ Charlie Gosse $\mathbb{D},^{5}$ Mircea Cotlet $\mathbb{D}^{6},{ }^{6}$ Oleg Gang $\left(\mathbb{D},{ }^{6,7}\right.$ Valérie Marchi $\left(\mathbb{D},^{2}\right.$ \\ and Franck Artzner (iD ${ }^{1}$
}

\author{
${ }^{1}$ Université de Rennes 1, CNRS UMR 6251, Institut de Physique de Rennes, Avenue du Général Leclerc, 35042 Rennes, France \\ ${ }^{2}$ Université de Rennes 1, CNRS UMR 6226, Institut des Sciences Chimiques de Rennes, Avenue du Général Leclerc, \\ 35042 Rennes, France \\ ${ }^{3}$ Synchrotron Soleil, L'Orme des Merisiers, Saint-Aubin BP 48, 91192 Gif-sur-Yvette, France \\ ${ }^{4}$ Laboratorio Nacional de Luz Síncrotron, CEP 13083-970, Caixa, Postal 6192 Campinas, São Paulo, Brazil \\ ${ }^{5}$ Laboratoire de Photonique et de Nanostructures, LPN-CNRS, Route de Nozay, 91460 Marcoussis, France \\ ${ }^{6}$ Center for Functional Nanomaterials, Brookhaven National Laboratory, Upton, New York 11973, USA \\ ${ }^{7}$ Department of Chemical Engineering and Department of Applied Physics and Applied Math Ematics, Columbia University, \\ New York, New York 10027, USA
}

\begin{abstract}
Correspondence should be addressed to Valérie Marchi; valerie.marchi@univ-rennes1.fr and Franck Artzner; franck.artzner@univ-rennes1.fr
\end{abstract}

Received 8 September 2021; Revised 13 October 2021; Accepted 26 October 2021; Published 24 November 2021

Academic Editor: Dong Kee Yi Copyright @ 92021 Thomas Bizien et al. This is an open access article distributed under the Creative Commons Attribution License,
which permits unrestricted use, distribution, and reproduction in any medium, provided the original work is properly cited.

\begin{abstract}
A specific organization of optically active nanoscale objects can greatly affect the optical response of a system. Here, we report the controlled modification of the fluorescent emission by the assembly of water-soluble quantum rods (QRs). Our study combines optical, electron microcopy, and X-ray scattering characterizations to reveal a correlation between the self-assembly behavior of QRs into ordered 3D-arrays and the optical properties (luminescence) of formed assemblies, where the observed optical response is highly dependent on the QR aspect ratio. Specifically, shorter, $18 \mathrm{~nm}$ long $\mathrm{QRs}\left(\mathrm{QR}_{18}\right)$, exhibiting a well-defined smectic packing, demonstrate an enhancement of the emission intensity accompanied by a red shift and a lifetime reduction. In contrast, $40 \mathrm{~nm}$ long $\mathrm{QRs}\left(\mathrm{QR}_{40}\right)$, forming a columnar phase, does not show these optical properties.
\end{abstract}

\section{Introduction}

Assembling colloids, thanks to bottom-up approaches offers an effective way to create a wide range of new materials with specific properties that emerge from the organization of uniform objects into structures periodic at all scales [1-4]. Such realizations are nowadays possible because of the diversity and the versatility of the method for most of the preparation of the nanoparticles with a high level of control over their size and shape, as well as their assembly in well-ordered superstructures [4-10]. In particular, quantum dots (QDs) are spherical colloids made of semiconductor materials, 2 to $10 \mathrm{~nm}$ in size, which exhibit a robust, efficient, and spectrally well-defined fluorescence emission [4-7]. Taking advantage of these remarkable fluorescence properties and of the opportunities offered by superstructures, many applications in the field of electronics and photonics have emerged, from lasing [8-12] to light harvesting devices [ 13, 14], and new physical effects have been evidenced, from fluorescence intensity enhancement to superradiance [15-18].

The optoelectronics properties of self-assembled QDs derive from a range of near-field interactions including energy 


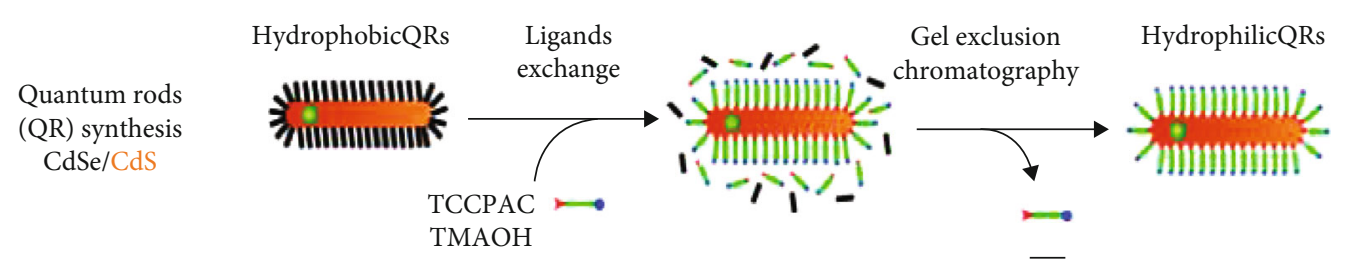

(a)

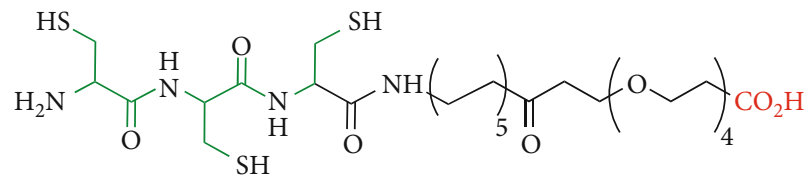

TCCPAC

(b)

FIgure 1: Preparation of the CdSe/CdS QRs. (a) Schematic route to the synthesis of hydrophobic quantum rods in suspension in toluene $\left(\mathrm{QR}^{\text {Toluene }}\right)$ and to their transformation, through ligand exchange, in hydrophilic quantum rods in suspension in water $\left(\mathrm{QR} \mathrm{R}^{\mathrm{Water}}\right)$. (b) Molecular formula of the TCCPAC peptidic ligand used for the exchange.

transfer [19] and electromagnetic field enhancement. Thus, they are directly affected by structural and electrostatic parameters such as the strength of the interparticle Coulomb interactions, the dielectric constant of the material, the topology, and the morphology of the assembly $[11,12]$. Among these parameters, the interparticle distance has tremendous effect on the optical coupling between neighboring nanoparticles [13]. In particular, due to the exciton coupling between proximal QDs, the fluorescence emission intensity can either increase or decrease, and the maximum wavelength of emission can also be shifted. $[15,19,20]$ The coupling of QD and plasmonic nanoparticles can also alter their intensity and fluorescent lifetime as well as modulate their polarization properties [1, 22, 23].

Many strategies have been developed to organize QDs [1, 24-26] into 2D and 3D higher-order architectures of wellcontrolled topology. One of the most efficient method to adjust the interdistance makes use of molecular recognition between biomolecular objects that are grafted on the nanoparticles, for instance DNA $[27,28]$ or proteins $[21,29,30]$. Another strategy is to exploit electrostatic interactions between protein filaments [31] and/or membranes [32] to generate 3D templates with monodisperse repeat distance in which the nanoparticles can fit. A strong increase in fluorescence intensity, as well as a bathochromic shift of the emission maximum, has already been observed in some of these assemblies [31].

Besides such biomimetic approaches, a simple strategy is to harness the tendency of anisotropic nanoparticles to selfassemble into 3D-arrays, herein called supracrystals. More specifically, as predicted by Onsager's theory [23, 24], it has been demonstrated that anisotropic semiconductor nanocrystals, also named quantum rods (QR), exhibit a rich polymorphism, which includes nematic and smectic liquid crystal phases [10, 33-35]. Previously, we have described a simple, yet powerful, strategy for shaping self-assemblies of water-soluble rod-like nanoparticles, either gold nanorods [36] or QRs [37]: an aqueous suspension is imprinted between a flat substrate and a topologically micropatterned mold, and, after water has evaporated, walls displaying a smectic B packing are obtained, organized on a regular lattice over several square millimeters. On the basis of this work, we here more deeply report on the influence of a variation of the QR aspect ratio on the structural properties of the obtained supracrystals. Furthermore, we characterized the fluorescence emission properties of the obtained assemblies and found that $\mathrm{QR}$ organization into 3D-ordered smectic crystal induced a red shift of the wavelength and an enhancement of the intensity of the fluorescence emission as well as a reduction of its lifetime.

\section{QRs Synthesis and Morphological Characterization}

Starting from the same batch of quasispherical CdSe seeds, we synthesized hydrophobic QRs of different lengths using a slightly modified version $[37,38]$ of the protocol reported by Carbone et al. [39, 40] (see Supporting Information for details). These QRs are obtained solubilized in toluene and are stabilized by a mixture of trioctyl phosphine oxide (TOPO), octadecylphosphonic acid (ODPA), and hexylphosphonic acid (HPA) (Figure 1(a)). Two batches were characterized by transmission electron microscopy (TEM). Both particle mean diameters, 2 $r_{\mathrm{QR}}$, were found around $6 \mathrm{~nm}$, and the length was 18 and $40 \mathrm{~nm}$ (Figure S1); thus, we called them $\mathrm{QR}_{18}^{\text {Toluene }}$ and $\mathrm{QR}_{40}^{\text {Toluene }}$, respectively. For further solubilization in water, the hydrophobic QRs were first incubated in the presence of an aqueous solution of the TCCPAC peptide (Figure 1(b)) also containing tetramethylammonium hydroxide. Ligands (TOPO, ODPA, HPA) are exchanged for TCCPAC, and the QRs dissolve into the aqueous phase. The resulting hydrophilic QRs were finally purified by gel exclusion chromatography to remove the ligands in excess, and it yielded two samples that we, respectively, denoted $\mathrm{QR}_{18}^{\text {Water }}$ and $\mathrm{QR}_{40}^{\text {Water }}$.

Upon transfer from the organic phase to the aqueous one, both batches of QRs exhibited a slight red shift of their maximum of fluorescence emission intensity, from 581 to $584 \mathrm{~nm}$. A typical evolution of the fluorescence spectra exhibits a small decrease in their emission quantum yield, from 0.55 to 0.40 as shown in Fig.S2a for $\mathrm{QR}_{18}$ and similar fluorescence emission decay (Fig.S2b, Table S1A). The fluorescent emission red shift may be attributed to the ligand tricystein interaction with the surface $\mathrm{Zn}$ and $\mathrm{S}$ atoms $[32,41,42]$ and the solvent change. 

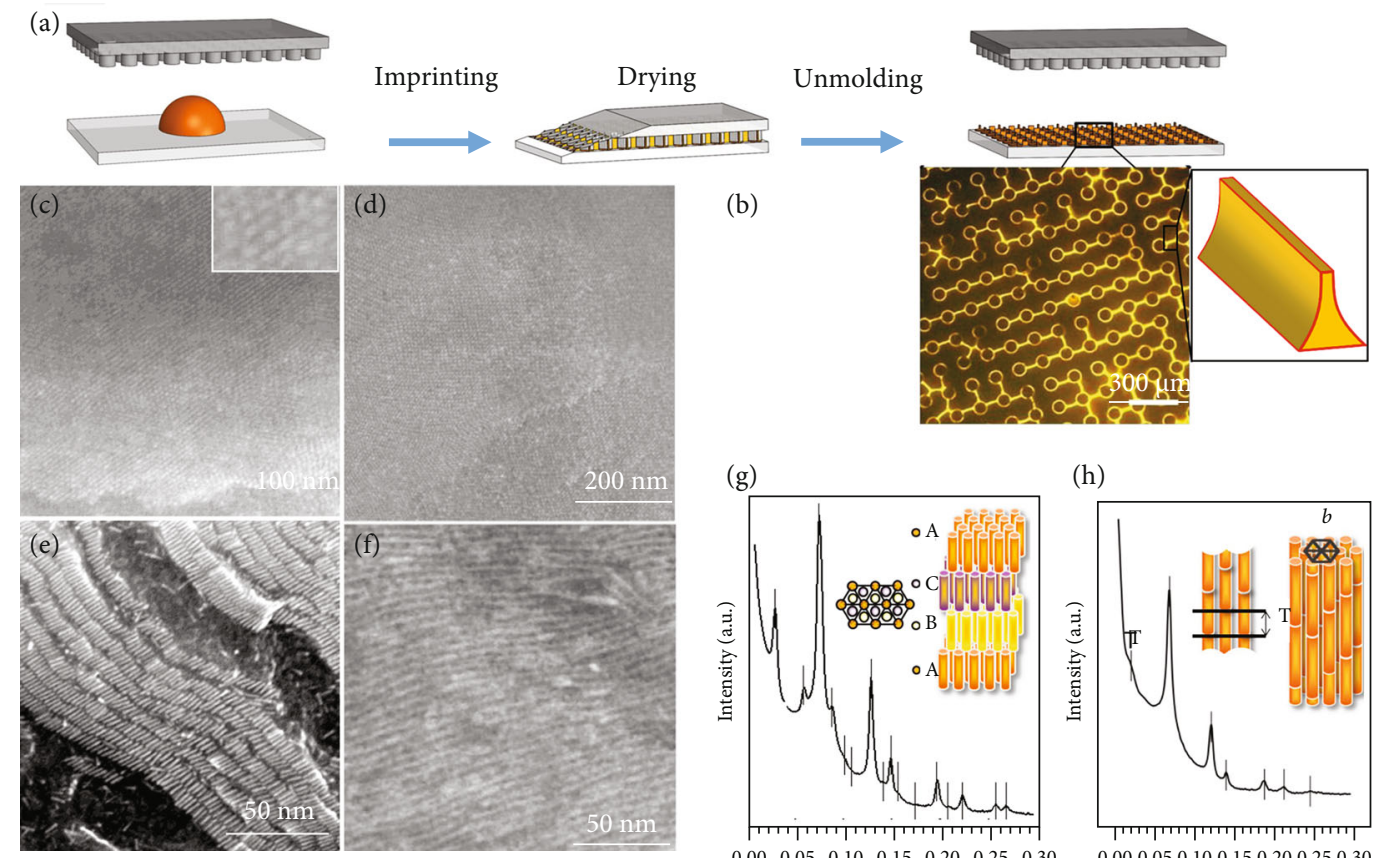

(g)

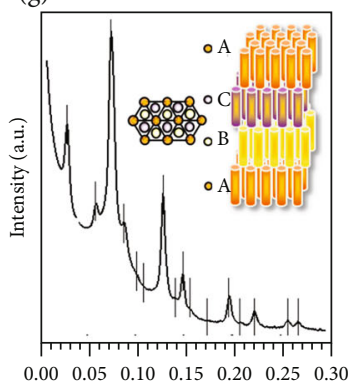

$\mathrm{q}\left(\mathrm{A}^{-1}\right)$ (h)

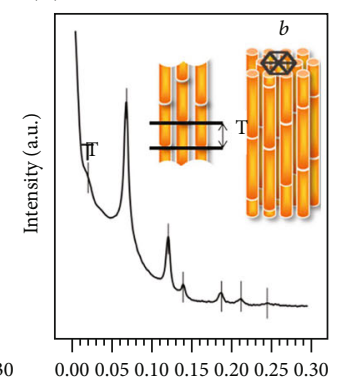

$q\left(A^{-1}\right)$

Figure 2: Preparation of the CdSe/CdS QR supracrystals and determination of their structures. (a) Schematic view of the controlled drying process, between a silicon wafer and a microstructured PDMS stamp that allows one to obtain $\mathrm{QR}^{\text {Pattern }}$ samples from aqueous $\mathrm{QR}^{\text {Water }}$ suspensions. (b) Fluorescence image of the walls observed for $\mathrm{QR}_{18}^{\text {Pattern }}$. (c) SEM image of the surface of a $\mathrm{QR}_{18}^{\text {Pattern }}$ wall and zoom on a surface showing the QR hexagonal ordering (the bare in zoom represents $10 \mathrm{~nm}$ ). (d) Similar observations performed on $\mathrm{QR}_{40}^{\text {Pattern }}$. (e) SEM image of a crack present on a $\mathrm{QR}_{18}^{\text {Pattern }}$ wall after cleavage showing the in-plane lateral smectic ordering. (f) Similar observation performed on $\mathrm{QR}_{40}^{\text {Pattern }}$. (g) SAXS intensity plot acquired on $\mathrm{QR}_{18}^{\text {Capillary }}$. Theoritical peak positions for a smectic $\mathrm{B}$ phase displaying an ABC packing with lattice parameters $a=9.76 \mathrm{~nm}$ and $c=63.8 \mathrm{~nm}$ are indicated (see Tab. S3 for full indexation). (h) SAXS intensity plot acquired on $\mathrm{QR}_{40}^{\text {Capillary }}$. Theoritical peak positions for a hexagonal columnar phase with a half $\mathrm{QRs}$ length shift between each column and with a lattice parameter $a=9.75 \mathrm{~nm}$, are indicated (see Tab. S4 for full indexation).

\section{QR Supracrystals Synthesis and Morphological Characterization}

We next focused on obtaining long-range order in 3D colloidal crystals having a size of at least several tens of microns. To do so, we used a previously developed approach in which we let dry an aqueous suspension of QRs in between a flat substrate, either a silicon wafer or a glass coverslip, and a PDMS stamp on which round pillars, $35 \mu \mathrm{m}$ high and $100 \mu \mathrm{m}$ in radius, are regularly spaced by $75 \mu \mathrm{m}$ on a square lattice (Figure 2(a)) [36, 43]. More precisely, sample $\mathrm{QR}_{18}^{\text {Pattern }}$ was prepared from $\mathrm{QR}_{18}^{\text {Water }}$ at $2.5 \mu \mathrm{M}$ supplemented by Tween 20 at $96.4 \mu \mathrm{M}$ whereas sample $\mathrm{QR}_{40}^{\text {Pattern }}$ was similarly obtained starting from a solution of $\mathrm{QR}_{40}^{\text {Water }}$ (Table S2A). In both cases, after unmolding, one could observe fluorescent walls forming a maze-like pattern on the substrate. These tridimensional walls connect the positions previously occupied by the pillars and typically have a triangular section in the range of 10 to $20 \mu \mathrm{m}$ (Figure 2(b)).

Studying the internal organization of such self-assembled supracrystals is essential for understanding the relationship between 3D structuration and emergent optical properties. First, the micropatterns made of QRs were observed by scan- ning electron microscopy (SEM). Sample cleavage cracked the walls, thereby opening the windows through which one could gain insight into colloidal packing. In some of the crevices obtained on both $\mathrm{QR}_{18}^{\text {Pattern }}$ and $\mathrm{QR}_{40}^{\text {Pattern }}$, dots arranged on a hexagonal lattice were observed (Figures 2(c) and 2(d), respectively). In some other crevices, it was also possible to observe nanoparticles from the side, and here, the two structures differed markedly. Whereas in $\mathrm{QR}_{18}^{\text {Pattern }}$, rods are clearly longitudinally organized in lamellae (Figure $2(\mathrm{e})$ ), in $\mathrm{QR}_{40}^{\text {Pattern }}$, one could only view surfaces covered with long and parallel linear motifs (Figure 2(f)).

Yet, SEM can only provide information on the inner structure of the supracrystals through cracks. It is also difficult to obtain precise lattice parameters from such measurements. Thus, small-angle X-ray scattering (SAXS) was used to overcome these limitations and investigate the bulk of $\mathrm{QR}$ assemblies. An aqueous $\mathrm{QR}_{18}^{\text {Water }}$ suspension was poured into a glass capillary and slowly dried under vacuum, so as to yield the $\mathrm{QR}_{18}^{\text {Capillary }}$ sample after one week (see Supporting Information for details). With a beam size smaller than any of the capillary dimensions, it was first possible to check for polymorphism within the sample: none was found since all the recorded diffraction patterns were totally superimposable (Fig. S4). The formed supracrystal could next be 
indexed as a smectic $B(\mathrm{SmB})$ phase with an $\mathrm{ABC}$ packing (Figure 2(g)). The unit cell parameters are $a=97.6 \AA$ and $c=638 \AA$, which correspond to a lateral hexagonal spacing $a_{\mathrm{SmB}}=a=97.6 \AA$ and to a lamellar repeat distance $d_{\mathrm{SmB}}=c l$ $3=212.6 \AA$ (see Tab. S3 for full indexation). As a matter of fact, SAXS results fully support SEM observations although preparation conditions were slightly different. Indeed, Tween 20 was added in the starting solution used for the microstructured patterns. As a control, we recorded the SAXS intensity plot in the presence of detergent, and no change could be noted (Fig. S5), proving that this compound does not influence $\mathrm{QR}$ packing.

In the case of the $\mathrm{QR}_{40}^{\text {Capillary }}$ sample, which was prepared in the same manner as sample $\mathrm{QR}_{18}^{\text {Capillary }}$ but starting from $\mathrm{QR}_{40}^{\text {Water }}$, SAXS data show that the structure obtained after drying can be indexed as a hexagonal columnar phase with a unit cell parameter $b=99.7 \AA$ (Figure $2(\mathrm{~h}$ ), and Tab. S4 for full indexation). Once more, $\mathrm{X}$-ray scattering measurements agree with SEM observations. Additionally, the shoulder named $\mathrm{T}$ gives an interesting detail about the columnar arrangement, which could not be evidenced by SEM. By analogy to what we have previously observed on gold nanorods columnar phases [36], it suggests that some QR columns are shifted by half of a nanoparticle length with respect to the adjacent columns.

Such smectic/columnar polymorphism of nanorods is usual and has been observed in the case of goethite inorganic materials [44], and of cylindrical viruses [45]. Both polydispersity and repulsion interaction features are invoked to impose the packing at the thermodynamical equilibrium. Lateral interactions are similar in both $\mathrm{QR}_{18}$ and $\mathrm{QR}_{40}$. However, the relative polydispersity (Fig. S1) of $\mathrm{QR}_{18}$ is higher than $\mathrm{QR}_{40}$. By analogy, goethite nanorods could be the origin of the observed smectic ordering.

To summarize our structural analyses, we can say that the surface chemistry of the functionalized, water-soluble QRs and the use of a microstructured stamp make possible to form supracrystals with a well-defined positional and orientational smectic order at a large scale in the case of $\mathrm{QR}_{18}$ and a columnar type order for $\mathrm{QR}_{40}$. In addition, the good quality of the long-range ordering could not be achieved with $\mathrm{QR}_{18}$. Importantly, whereas the surface-to-surface distances are in the same range, $\approx 3-4 \mathrm{~nm}$ for both $\mathrm{QR}_{18}$ and $\mathrm{QR}_{40}$, the center-to-center distance is much smaller and uniform for $\mathrm{QR}_{18}$, owing to the nature of the smectic $\mathrm{B}$ arrangement with respect to the one of a columnar hexagonal one. Indeed, for $\mathrm{QR}_{40}$, the center-to-center distance is on average longer (due to the higher aspect ratio and displacement in columnar state) and therefore varies a lot due random vertical shift. In the following, the $\mathrm{QR}_{40}$ will consequently be used as a control.

\section{QR Supracrystals Fluorescence Emission Properties}

In order to correlate the organization of the emitters with the optical properties of their assemblies, we recorded the fluorescence emission spectra and the fluorescence emission decay curves of the two hydrophilic QR batches in two distinct condensed, dried states. The first one, $\mathrm{QR}^{\text {Pattern }}$, corresponds to the microstructured supracrystals described above and measurements with a confocal setup have been performed in the upper part of the walls presented in Figure 2(b). The second type of condensed state, $\mathrm{QR}^{\text {Heap }}$, corresponds to aggregates resulting from an undirected drying process without the PDMS stamp. In such aggregates, nanoparticles locally display a crystal liquid order, the phasing identical to the one observed in the capillary and in the walls. Finally, since for both QR lengths, sample preparation proceeded from nanoparticles dispersed at the same concentration in water $(2.5 \mu \mathrm{M})$, we can assume that the number density of emitters in a given condensed state is more or less the same, within experimental errors and within a factor $\approx 2$ that would account for the difference in volume between $\mathrm{QR}_{18}$ and $\mathrm{QR}_{40}$.

We first analyzed the fluorescence spectra of the condensed dried states and compare them to the spectra obtained from noninteracting $\mathrm{QR}^{\text {Water }}$ in an aqueous suspension. We noted that the ratio of emission intensities at $\lambda_{\max }$ between $\mathrm{QR}_{18}^{\text {Heap }}$ and $\mathrm{QR}_{40}^{\text {Heap }}$ is equal to 2.5 (Figures 3(a) and 3(c), green curves), which can be interpreted as a consequence of a roughly twice higher density of emitters for the sample made of roughly twice shorter nanoparticles. In contrast, the ratio of emission intensities at $\lambda_{\max }$ between $\mathrm{QR}_{18}^{\text {Pattern }}$ and $\mathrm{QR}_{40}^{\text {Pattern }}$ is equal to 190 (Figures 3(a) and 3(c), gray curves). Furthermore, with a $9 \mathrm{~nm}$ bathochromic shift, the $\mathrm{QR}_{18}^{\text {Pattern }}$ sample is the only one that displays a maximum intensity wavelength different from the $583-584 \mathrm{~nm}$ value (Table 1 ).

Now considering the fluorescence emission decay curves, it can be observed that nanoparticles in dried states display shorter average lifetimes $\left(\tau_{a v}=2-6 \mathrm{~ns}\right)$ in comparison to the noninteracting $\mathrm{QR}^{\text {Water }}$ in water $\left(\tau_{a v}=14 \mathrm{~ns}\right)$. In the condensed dried states, the contribution of the larger lifetime strongly decreases, whereas the ones of the two smaller lifetimes increase (see $\mathrm{QR}^{\text {Pattern }}$ and $\mathrm{QR}^{\text {Heap }}$ in Table 1 and Figures 3(b) and 3(d)). To be more precise, in $\mathrm{QR}^{\text {Heap }}$ aggregates, the values of the preexponential factors are in the range of $A_{1}=5, A_{2}=23-30$, and $A_{3}=65-72 \%$ for both QR lengths resulting in an average lifetime $\tau_{a v}=1.9-2.1$ ns. The $\mathrm{QR}_{18}^{\text {Pattern }}$ microstructured supracrystals are characterized by similar figures $\left(A_{1}=5, A_{2}=22-30\right.$, and $A_{3}=73$ $\%)$ with an average lifetime $\tau_{a v}=1.6 \mathrm{~ns}$, but for $\mathrm{QR}_{40}^{\text {Pattern }}$, the decrease in lifetime is less marked $\tau_{a v}=5.9 \mathrm{~ns}$ with preexponential factors values $A_{1}=18, A_{2}=51$, and $A_{3}=31 \%$.

These experiments show three specific photophysical

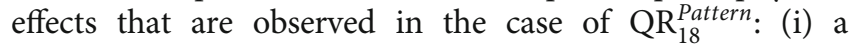
decrease in lifetime accompanied by (ii) an enhancement of the emission intensity with (iii) a $9 \mathrm{~nm}$ bathochromic shift. It is worthwhile to discuss if the particular behavior of $\mathrm{QR}_{18}^{\text {Pattern }}$ could be directly related to the smectic order present in the supracrystals, for which both the in-plane and the normal organization of QRs might favor an efficient long range optical coupling.

The observed fluorescence exaltation is an increase of the emitted photons per time-unit which can find origin either in a nonradiative process vanishing or in a photon emission 


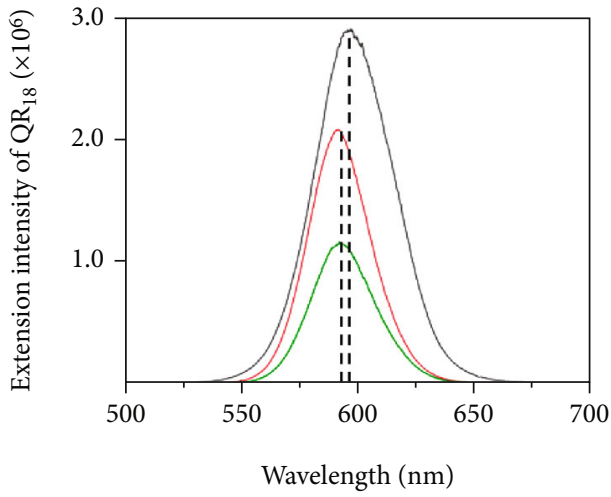

(a)

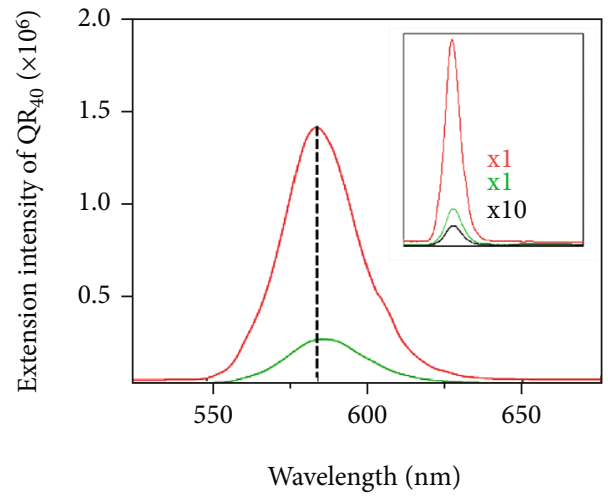

(c)

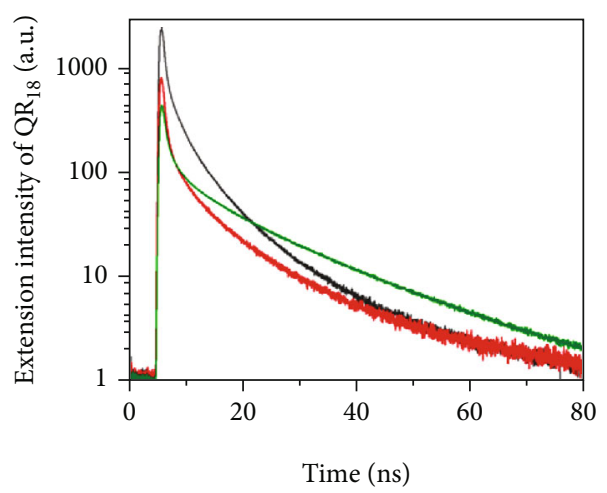

(b)

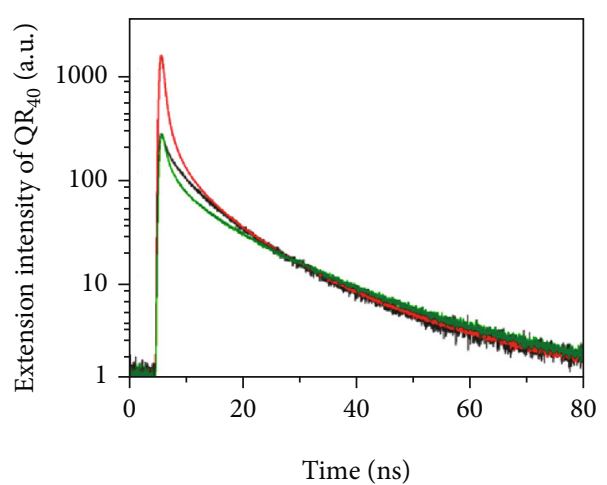

(d)

FIgURE 3: Fluorescence emission properties of CdSe/CdS QRs interacting with each other in various dried states. The nanoparticles are either part of supracrystals $\left(\mathrm{QR}^{\text {Pattern }}\right.$ in grey), obtained by letting the droplet dry in between a coverslip and a microstructured PDMS stamp (see Figure 2), or part of aggregates with local liquid crystal order $\left(\mathrm{QR}^{\text {Heap }}\right.$ in green), obtained without stamp. Emitters dispersed in water at $2.5 \mu \mathrm{M}\left(\mathrm{QR}^{\text {Water }}\right.$ in red) are used as references since they correspond to noninteracting nanoparticles. Measurements were all performed on the confocal setup, and the excitation wavelength was set at $\lambda_{\text {exc }}=400 \mathrm{~nm}$. (a) Fluorescence emission spectra for $18 \mathrm{~nm}$ long quantum rods $\left(\mathrm{QR}_{18}\right)$ for the two dried samples, $\mathrm{QR}_{18}^{\text {Pattern }}$ (gray) and $\mathrm{QR}_{18}^{\text {Heap }}$ (green), and for the aqueous suspension, $\mathrm{QR} \mathrm{R}_{18}^{\mathrm{Water}}$. (b) Corresponding fluorescence emission decay curves. (c) Fluorescence emission spectra for $40 \mathrm{~nm}$ long quantum rods $\left(\mathrm{QR}_{40}\right)$ for the two dried samples, $\mathrm{QR}_{40}^{\text {Pattern }}$ (gray) and $\mathrm{QR}_{40}^{\text {Heap }}$ (green), and for the aqueous suspension, $\mathrm{QR}_{40}^{\text {Water }}$. The $\mathrm{QR}_{40}^{\text {Pattern }}$ fluoresence emission spectrum was almost superimposed with the baseline, so it was magnified by a factor 10 in the insert for sake of clarity. (d) Corresponding fluorescence emission decay curves.

TABLE 1: Influence of the QR ordering on the maximum intensity wavelength $\left(\lambda_{\max }\right)$ and on the decay of fluorescence emission for 18 and $40 \mathrm{~nm}$ long CdSe/CdS QRs (see Figure 3 for detailed experimental conditions and graphical data). Deconvolution of fluorescence emission decay curves using three exponentials yields (see Fig. S3), for each condition and each species, three preexponential factors, $A_{i}$, and three lifetimes, $\tau_{i} . \tau_{\text {av }}$ designed the average lifetime of the $\tau_{i}$. "Water" corresponds to measurements on QRs in aqueous solution whereas all the other conditions refer to dried states. In "Heap," they lay aggregated with a local liquid crystal order, and finally in "Pattern," they are organized in microstructured supracrystals.

\begin{tabular}{|c|c|c|c|c|c|c|c|c|}
\hline Sample & $\lambda_{\max }(\mathrm{nm})$ & $A_{1}(\%)$ & $\tau_{1}(\mathrm{~ns})$ & $A_{2}(\%)$ & $\tau_{2}(\mathrm{~ns})$ & $A_{3}(\%)$ & $\tau_{3}(\mathrm{~ns})$ & $\tau_{a v}(\mathrm{~ns})$ \\
\hline $\mathrm{QR}_{18}^{\text {Water }}$ & 584 & 72 & 18.7 & 19 & 5.1 & 9 & 0.8 & 14.5 \\
\hline $\mathrm{QR}_{18}^{\text {Heap }}$ & 584 & 5 & 13.3 & 30 & 2.8 & 65 & 0.6 & 1.9 \\
\hline $\mathrm{QR}_{18}^{\text {Pattern }}$ & 593 & 5 & 11.1 & 22 & 3.2 & 73 & 0.5 & 1.6 \\
\hline $\mathrm{QR}_{40}^{\text {Water }}$ & 583 & 71 & 18.5 & 15 & 4.0 & 14 & 0.6 & 13.8 \\
\hline $\mathrm{QR}_{40}^{\text {Heap }}$ & 583 & 5 & 16.7 & 23 & 3.2 & 72 & 0.7 & 2.1 \\
\hline $\mathrm{QR}_{40}^{\text {Pattern }}$ & 583 & 18 & 15.9 & 51 & 5.4 & 31 & 0.8 & 5.9 \\
\hline
\end{tabular}


turnover increase. Indeed, an excited molecule or nanostructure relaxes to a lower energy state through radiative emission of a photon and nonradiative processes can depopulate the excited state, with respective decay rates $k_{\text {rad }}$ and $k_{\text {nrad }}$. The emission flux is proportional to the quantum yield $\Phi=k_{\text {rad }} /\left(k_{\text {rad }}+k_{\text {nrad }}\right)$ and can increase through an increase of $k_{\text {rad }}$ or a decrease of $k_{\text {nrad }}$. The mean fluorescence lifetime is $\tau_{\mathrm{rad}}=1 / k_{\mathrm{rad}}$. Observing decrease concomitantly with an increase of quantum yield shows that the core mechanism is a reduction of the radiative lifetime. These observations are in lines with the ones reported in the literature with other types of nanoparticles superlattices [29, 46, 47]. This kind of photophysical behavior is rare and mostly related to collective effects. This can be enlightening by noting that similar results are observed for strongly coupled such as gas or dyes [48-51] and are attributed to superradiance or superfluorescence. Since no coherence has been observed, we should invoke an energy transfer that might be either by tunnel effect (such as FRET) or by dipolar electromagnetic interactions (such as superradiance). The smallest interdistance between QR surfaces $\left(c-2 r_{\mathrm{QRs}}\right)$ is large (here around $3.7 \mathrm{~nm}$ in the hexagonal lattice). For such distance, QR excited states do hardly interact via the tunnel effect [50]. In the present case, dipolar interaction must generate a cascade of coherent fluorescence called superradiance [48-52]. Such radiation reaction is accompanied by a significant energy loss, e.g., a slight red shift [53]. It has to be noticed that fluorescence intensity varies quadratically as $N^{2}, N$ being the number of coherent emission dipoles. In the present case, efficient absorber was required with $\mathrm{QR}_{18}^{\text {Pattern }}$ in order to not saturate the PM. Noteworthy, the superradiance is very sensitive to the positional order of the emiters due to the factor of interference between emission waves (Equation (1)).

$$
\Gamma\left(\vec{k}-\overrightarrow{k_{0}}\right)=\left|\frac{1}{N} \sum_{l=1}^{N} e^{i\left(\vec{k}-\vec{k}_{0}\right) \cdot \vec{r}_{l}}\right|^{2}
$$

This pair-interference term can be compared to the interference term for X-ray scattering. In our case, they are identical. This means that the pair-interference terms are exalted when absorption of dipoles are located in a $3 \mathrm{D}$ crystal. As mentioned, superradiance [48] does not correspond to any tunnel transfer of fluorescence such as in FRET. The excited states relax via dipolar interactions more efficiently in presence of an electromagnetic wave oscillating at a frequency close the emission energy difference, like in NMR. The emitted photon has energy close to the initial but is not coherent, neither in energy nor in direction. Such radiation reaction is accompanied by a slight bathochromic shift [31] which could be in agreement with the spectra we have recorded on $\mathrm{QR}_{18}^{\text {Pattern }}$. In the case of $\mathrm{QR}^{\text {Heap }}$, we do not observe any enhancement of the emission intensity regardless of the lengths of the nanoparticles, which we attribute to the smaller scale over which they are organized. For $\mathrm{QR}_{40}^{\text {Pattern }}$, the nanoparticles are ordered on a larger scale but their respective lateral positions are less controlled than in the smectic phase characterizing
$\mathrm{QR}_{18}^{\text {Pattern }}$. We evidenced the sensitivity of collective optical properties to the $3 \mathrm{D}$ positional order by the comparison between $\mathrm{QR}_{18}$ and $\mathrm{QR}_{40}$. The former sample is well organized in a $3 \mathrm{D}$ crystal and exhibits a fluorescence increase with a bathochromic shift linked to a lifetime reduction, while the latter is organized only in 2D and exhibits fluorescence properties similar to the individual QRs.

\section{Conclusion}

Our bottom-up strategy enables to self-organize in hierarchical organization: nanoparticles obey a 3D positional and orientational order over hundreds of nanometers, they are part of supracrystal having the shape of walls with a triangular crosssection a few tens of microns large, and these walls are patterned on a substrate at the millimeter scale. More specifically, a smectic $\mathrm{B}(\mathrm{ABC})$ phase is obtained with $18 \mathrm{~nm}$ long QRs and a hexagonal columnar phase is obtained with $40 \mathrm{~nm}$ long QRs, which reveal that the nanoparticle length can be used to tune the structural properties of the assemblies, and consequently their optical ones. Indeed, confocal measurements of the fluorescence emission spectra and decay curves have revealed that, although in the two supracrystals emission is faster than for noninteracting shorter species, and the most organized, it is the only one that displays a strong enhancement of the emitted intensity. Thus, our bottom-up approach could be of interest in the field of photonics, in order to simply realize optical systems with strong fluorescence quantum yield.

\section{Data Availability}

Data in supplementary information files (included).

\section{Disclosure}

Elsa Mazari-Arrighi's present address is CYTO/PCV/BIG/ DRF/CEA, Unité de Thérapie Cellulaire, Institut Universitaire d'Hématologie, Hôpital Saint Louis, 1 avenue Claude Vellefaux, 75010 Paris, France. Christophe Dupuis' present address is Centre de Nanosciences et de Nanotechnologies, UMR 9001 CNRS/Université Paris-Saclay, 10 boulevard Thomas Gobert, 91120 Palaiseau, France. Charlie Gosse's present address is Institut de Biologie de l'Ecole Normale Supérieure, ENS, CNRS, INSERM, PSL Research University, 46 rue d'Ulm, 75005 Paris, France.

\section{Conflicts of Interest}

The authors declare that they have no conflicts of interest.

\section{Authors' Contributions}

Thomas Bizien is responsible for synthesis and structural and optical measurements. Marie Postic is responsible for surface deposition of nanoparticles. Pascale Even-Hernandez is responsible for supervision of the QR colloidal characterization. Pascal Panizza is responsible for process of surface deposition. Cristelle Mériadec is responsible for SAXS experiments. Florian Meneau is responsible for SAXS experiments. Elsa Mazari-Arrighi is responsible for SEM analysis. Christophe 
Dupuis is responsible for SEM measurements. Charlie Gosse is responsible for supervision of SEM analysis and manuscript writing. Mircea Cotlet is responsible for supervision of the optical properties and lifetime particle measurements. Oleg Gang is responsible for structural analysis. Valérie Marchi is responsible for manuscript writing and planning of the $\mathrm{QR}$ synthesis and characterization. Franck Artzner is responsible for supervision of the project, the structural analysis and of the optical properties.

\section{Acknowledgments}

The Agence Nationale de la Recherche (ANR contract No. 16CE09-0027-02 HYPNAP and contract No. 14-CE08-0004 ARTEMIS) funded this work. We are indebted to the Region Bretagne, the French Direction Generale de l'Armement, and the ANR for the $\mathrm{PhD}$ financial supports to T. Bizien, M. Postic, and E. Mazari-Arrighi. We also acknowledge Université de Rennes for a travel grant to T. Bizien, so as to enable him to perform measurements at the Center for Functional Nanomaterials of the Brookhaven National Laboratory and Rennes Métropoles for equipment support. Oleg Gang was supported by the US Department of Defense, Army Research Office, grant W911NF-19-1-0395. This research used resources of the Center for Functional Nanomaterials, supported by U.S. DOE Office of Science Facilities at Brookhaven National Laboratory under contract No. DE-SC0012704.

\section{Supplementary Materials}

Experimental details are given about synthesis of the CdSe/CdS hydrophobic nanorods, transmission electron microscopy, UVVis absorption spectroscopy, quantum yield determination preparation of the "QR" supracrystals, scanning electron microscopy, small angle X-ray scattering, and confocal fluorescence spectromicroscopy. Figure S1: diameters and lengths of the hydrophobic QRs measured by transmission electron microscopy (TEM). (a) $\mathrm{QR}_{18}^{\text {Toluene }}$. (b) $\mathrm{QR}_{40}^{\text {Toluene }}$. Figure $\mathrm{S} 2$ : Characterization of the fluorescence emission properties of the QRs dispersed in toluene and in water at $2.5 \mu \mathrm{M}$. Figure S3: deconvolution of the fluorescence emission spectra of hydrophilics QRs dissolved at $2.5 \mu \mathrm{M}$ in a drop of water placed over a coverslip. Figure S4: Detailed SAXS data acquired on $\mathrm{QR}_{18}^{\text {Capillary }}$. Figure S5: SAXS intensity plot acquired when drying proceeds. Table S1: influence of the ligand and solvent on the maximum intensity wavelength $\left(\lambda_{\max }\right)$ and on the decay of fluorescence emission for $18 \mathrm{~nm}$ long QRs. Table S2: influence of the length of hydrophilic QRs on their fluorescence emission spectra. Table S3: full indexation for the SAXS intensity plot measured on sample $\mathrm{QR}_{18}^{\text {Capillary }}$ (Figure 2(f)). Table S4: Full indexation for the SAXS intensity plot measured on sample $\mathrm{QR}_{40}^{\text {Capillary }}$ (Figure 2(g)). (Supplementary Materials)

\section{References}

[1] Y. Tian, J. R. Lhermitte, L. Bai et al., "Ordered threedimensional nanomaterials using DNA-prescribed and valence-controlled material voxels," Nature Materials, vol. 19, no. 7, pp. 789-796, 2020.
[2] A. Klinkova, R. M. Choueiri, and E. Kumacheva, "Self-assembled plasmonic nanostructures," Chemical Society Reviews, vol. 43, no. 11, p. 3976, 2014.

[3] A. O. Govorov, G. W. Bryant, W. Zhang et al., "Exciton-plasmon interaction and hybrid excitons in semiconductor-metal nanoparticle assemblies," Nano Letters, vol. 6, no. 5, pp. 984994, 2006.

[4] L. Wang, L. Xu, H. Kuang, C. Xu, and N. A. Kotov, "Dynamic nanoparticle assemblies," Accounts of Chemical Research, vol. 45, no. 11, pp. 1916-1926, 2012.

[5] B. H. Kim, M. J. Hackett, J. Park, and T. Hyeon, "Synthesis, characterization, and application of ultrasmall nanoparticles," Chemistry of Materials, vol. 26, no. 1, pp. 59-71, 2014.

[6] D. J. Norris and M. G. Bawendi, "Measurement and assignment of the size-dependent optical spectrum in CdSe quantum dots," Physical Review B, vol. 53, p. 16338, 1996.

[7] P. Reiss, "Synthesis of Semiconductor Nanocrystals in Organic Solvents," in In semiconductor nanocrystal quantum dots: synthesis, assembly, spectroscopy and applications, A. L. Rogach, Ed., pp. 35-72, Springer Vienna, Vienna, 2008.

[8] J. N. Cha, M. H. Bartl, M. S. Wong, A. Popitsch, T. J. Deming, and G. D. Stucky, "Microcavity lasing from block peptide hierarchically assembled quantum dot spherical resonators," Nano Letters, vol. 3, no. 7, pp. 907-911, 2003.

[9] O. V. Kozlov, Y.-S. Park, J. Roh, I. Fedin, T. Nakotte, and V. I. Klimov, "Sub-single-exciton lasing using charged quantum dots coupled to a distributed feedback cavity," Science, vol. 365, no. 6454, pp. 672-675, 2019.

[10] M. Zavelani-Rossi, M. G. Lupo, R. Krahne, L. Manna, and G. Lanzani, "Lasing in self-assembled microcavities of CdSe/ CdS core/shell colloidal quantum rods," Nanoscale, vol. 2, no. 6 , p. 931, 2010.

[11] A. C. Scofield, S.-H. Kim, J. N. Shapiro et al., "Bottom-up photonic crystal lasers," Nano Letters, vol. 11, no. 12, pp. 53875390, 2011.

[12] C. Grivas, C. Li, P. Andreakou et al., "Single-mode tunable laser emission in the single-exciton regime from colloidal nanocrystals," Nature Communications, vol. 4, no. 1, 2013.

[13] J. Liu, J. Mantell, N. D. Bartolo, and M. R. Jones, "Mechanisms of self-assembly and energy harvesting in tuneable conjugates of quantum dots and engineered photovoltaic proteins," Small, vol. 15, no. 4, p. 1804267, 2019.

[14] I. Robel, V. Subramanian, M. Kuno, and P. V. Kamat, "Quantum Dot Solar Cells. Harvesting Light Energy with CdSe Nanocrystals Molecularly Linked to Mesoscopic TiO2 Films," Journal of the American Chemical Society, vol. 128, no. 7, pp. 2385-2393, 2006.

[15] S. H. Ko, K. Du, and J. A. Liddle, "Quantum-dot fluorescence lifetime engineering with DNA origami constructs," Angewandte Chemie International Edition, vol. 52, no. 4, pp. 1193-1197, 2013.

[16] D. Englund, D. Fattal, E. Waks et al., "Controlling the spontaneous emission rate of single quantum dots in a 2D photonic crystal," Physical Review Letters, vol. 95, no. 1, article 013904, 2005.

[17] A. E. Krasnok, A. P. Slobozhanyuk, C. R. Simovski et al., “An antenna model for the purcell effect," Scientific Reports, vol. 5, no. 1, 2015.

[18] Z. Tang, "Spontaneous Organization of single CdTe nanoparticles into luminescent nanowires," Science, vol. 297, no. 5579, pp. 237-240, 2002. 
[19] S. A. Crooker, J. A. Hollingsworth, S. Tretiak, and V. I. Klimov, "Spectrally resolved dynamics of energy transfer in quantum-dot assemblies: towards engineered energy flows in artificial materials," Physical Review Letters, vol. 89, no. 18), 2002.

[20] J. J. Choi, J. Luria, B.-R. Hyun et al., "Photogenerated exciton dissociation in highly coupled lead salt nano-crystal assemblies," Nano Letters, vol. 10, no. 5, pp. 1805-1811, 2010.

[21] M. Fernandez, A. Urvoas, P. Even-Hernandez et al., "Hybrid gold nanoparticle-quantum dot self-assembled nanostructures driven by complementary artificial proteins," Nanoscale, vol. 12, no. 7, pp. 4612-4621, 2020.

[22] H. Zhang, M. Li, K. Wang et al., "Polarized single-particle quantum dot emitters through programmable cluster assembly," ACS Nano, vol. 14, no. 2, pp. 1369-1378, 2020.

[23] D. Ge, S. Marguet, A. Issa et al., "Hybrid plasmonic nanoemitters with controlled single quantum emitter positioning on the local excitation field," Nature Communications, vol. 11, no. 1, p. 3414, 2020.

[24] E. V. Shevchenko, D. V. Talapin, N. A. Kotov, S. O’Brien, and C. B. Murray, "Structural diversity in binary nanoparticle superlattices," Nature, vol. 439, no. 7072, pp. 55-59, 2006.

[25] M. A. Boles, M. Engel, and D. V. Talapin, "Self-assembly of colloidal nanocrystals: from intricate structures to functional materials," Chemical Reviews, vol. 116, no. 18, pp. 1122011289, 2016

[26] D. Sun and O. Gang, "Binary heterogeneous superlattices assembled from quantum dots and gold nanoparticles with DNA," Journal of the American Chemical Society, vol. 133, no. 14, pp. 5252-5254, 2011.

[27] D. Nykypanchuk, M. M. Maye, D. van der Lelie, and O. Gang, "DNA-guided crystallization of colloidal nanoparticles," Nature, vol. 451, no. 7178, pp. 549-552, 2008.

[28] S. Y. Park, A. K. R. Lytton-Jean, B. Lee, S. Weigand, G. C. Schatz, and C. A. Mirkin, "DNA-programmable nanoparticle crystallization," Nature, vol. 451, no. 7178, pp. 553-556, 2008.

[29] Z. Nie, A. Petukhova, and E. Kumacheva, "Properties and emerging applications of self-assembled structures made from inorganic nanoparticles," Nature Nanotech, vol. 5, no. 1, pp. 15-25, 2010.

[30] K. L. Gurunatha, A. C. Fournier, A. Urvoas et al., "Nanoparticles self-assembly driven by high affinity repeat protein pairing," ACS Nano, vol. 10, no. 3, pp. 3176-3185, 2016.

[31] E. Henry, A. Dif, M. Schmutz et al., "Crystallization of fluorescent quantum dots within a three-dimensional bio-organic template of actin filaments and lipid membranes," Nano Letters, vol. 11, no. 12, pp. 5443-5448, 2011.

[32] A. Dif, E. Henry, F. Artzner et al., "Interaction between watersoluble peptidic CdSe/ZnS nanocrystals and membranes: formation of hybrid vesicles and condensed lamellar phases," Journal of the American Chemical Society, vol. 130, no. 26, pp. 8289-8296, 2008.

[33] D. V. Talapin, R. Koeppe, S. Götzinger et al., "Highly emissive colloidal CdSe/CdS heterostructures of mixed dimensionality," Nano Letters, vol. 3, no. 12, pp. 1677-1681, 2003.

[34] I. C. Robin, R. André, A. Balocchi et al., "Purcell effect for CdSe/ZnSe quantum dots placed into hybrid micropillars," Applied Physics Letters, vol. 87, no. 23, p. 233114, 2005.

[35] R. Alam, D. M. Fontaine, B. R. Branchini, and M. M. Maye, "Designing quantum rods for optimized energy transfer with firefly luciferase enzymes," Nano Letters, vol. 12, no. 6, pp. 3251-3256, 2012.

[36] C. Hamon, M. Postic, E. Mazari et al., "Three-dimensional self-assembling of gold nanorods with controlled macroscopic shape and local smectic B order," ACSNano, vol. 6, no. 5, pp. 4137-4146, 2012.

[37] T. Bizien, P. Even-Hernandez, M. Postic et al., "Peptidic ligands to control the three-dimensional self-assembly of quantum rods in aqueous media," Small, vol. 10, no. 18, pp. 3707-3716, 2014.

[38] T. Bizien, J.-C. Ameline, K. G. Yager, V. Marchi, and F. Artzner, "Self-organization of quantum rods induced by lipid membrane corrugations," Langmuir, vol. 31, no. 44, pp. 12148-12154, 2015.

[39] L. Carbone, C. Nobile, M. De Giorgi et al., "Synthesis and micrometer-scale assembly of colloidal CdSe/CdS Nanorods prepared by a seeded growth approach," Nano Letters, vol. 7, no. 10, pp. 2942-2950, 2007.

[40] F. D. Stasio, J. Q. Grim, V. Lesnyak et al., "Single-mode lasing from colloidal water-soluble CdSe/CdS quantum dot-in-rods," Small, vol. 11, no. 11, pp. 1328-1334, 2015.

[41] J. M. Tsay, S. Doose, F. Pinaud, and S. Weiss, "Enhancing the photoluminescence of peptide-coated nanocrystals with shell composition and UV irradiation," The Journal of Physical Chemistry B, vol. 109, no. 5, pp. 1669-1674, 2005.

[42] F. Pinaud, D. King, H. P. Moore, and S. Weiss, "Bioactivation and cell targeting of semiconductor CdSe/ZnS nanocrystals with phytochelatin-related peptides," Journal of the American Chemical Society, vol. 126, pp. 6115-6123, 2004.

[43] P. Panizza, H. Algaba, M. Postic, G. Raffy, L. Courbin, and F. Artzner, "Order-disorder structural transitions in mazes built by evaporating drops," Physical Review Letters, vol. 121, no. 7, article 078002, 2018.

[44] G. . J. Vroege, D. . M. . E. Thies-Weesie, A. . V. Petukhov, B. . J. Lemaire, and P. Davidson, "Smectic liquid-crystalline order in suspensions of highly polydisperse goethite nanorods," Advanced Materials, vol. 18, pp. 2565-2568, 2006.

[45] E. Grelet and R. Rana, "From soft to hard rod behavior in liquid crystalline suspensions of sterically stabilized colloidal filamentous particles," Soft Matter, vol. 12, pp. 4621-4627, 2016.

[46] M. Scheibner, T. Schmidt, L. Worschech et al., "Superradiance of quantum dots," Nature Phys, vol. 3, no. 2, pp. 106-110, 2007.

[47] G. Raino, M. A. Becker, M. I. Bodnarchuk, F. R. Mahrt, M. V. Kovalenko, and T. Stoëferle, "Superfluorescence from lead halide perovskite quantum dot superlattices," Nature, vol. 563, pp. 671-675, 2018.

[48] R. H. Dicke, "Coherence in spontaneous radiation processes," Physical Review, vol. 93, no. 1, pp. 99-110, 1954.

[49] R. Monshouwer, M. Abrahamsson, F. van Mourik, and R. van Grondelle, "Superradiance and exciton delocalization in bacterial photosynthetic light-harvesting systems," The Journal of Physical Chemistry B, vol. 101, no. 37, pp. 7241-7248, 1997.

[50] T. Meier, Y. Zhao, V. Chernyak, and S. Mukamel, "Polarons, localization, and excitonic coherence in superradiance of biological antenna complexes," The Journal of Chemical Physics, vol. 107, no. 10, pp. 3876-3893, 1997.

[51] M. Gross and S. Haroche, "Superradiance: an essay on the theory of collective spontaneous emission," Physics Reports, vol. 93, no. 5, pp. 301-396, 1982. 
[52] A. R. Clapp, I. L. Medintz, J. M. Mauro, B. R. Fisher, M. G. Bawendi, and H. Mattoussi, "Fluorescence resonance energy transfer between quantum dot donors and dye-labeled protein acceptors," Journal of the American Chemical Society, vol. 126, no. 1, pp. 301-310, 2004.

[53] L. E. J. H. Allen, Optical Resonance and Two-Level Atoms, Dover, 1987. 